\title{
Confección de un prototipo de pantaleta con adaptación de compresas térmicas para
} disminución de cólicos menstruales

\section{Preparation of a prototype panties with adaptation of thermal pads to reduce menstrual cramps}

\author{
VÁZQUEZ-JUÁREZ, Yolanda*†, RAMÍREZ-MONDRAGÓN, Xóchitl y TENORIO-LARA, Raúl \\ Universidad Tecnológica del Suroeste de Guanajuato, carretera Valle-Huanímaro Km. 1.2, Valle de Santiago, Gto. México
}

ID 1 ${ }^{\text {er }}$ Autor: Yolanda, Vázquez-Juárez / ORC ID: 0000-0002-1571-2145

ID $1^{\text {er }}$ Coautor: Xóchitl, Ramírez-Mondragón / ORC ID: 0000-0003-2631-9039

ID $2^{\text {do }}$ Coautor: Raúl, Tenorio-Lara / ORC ID: 0000-0002-4390-3205

\section{Resumen}

Este trabajo muestra un el proceso de patronaje y confección un prototipo de pantaleta con adaptación de compresas térmicas para disminución de cólicos menstruales adaptado a las necesidades y requerimientos de las mujeres. Se habla de la transformación realizada para la confección de las prendas muestras, a partir de una plantilla básica, así como de los 2 procesos de ensamble de las misma y la maquinaría utilizada, del mismo modo se consideran las modificaciones realizadas a la transformación para mejor adaptación al cuerpo en cuanto al corte de a prenda y uso de materiales. Se muestran los resultados de la pantaleta con la adaptación de la bolsa para compresa en función a los materiales usados para dicho fin, donde se determino que lo más conveniente para el uso final de la prenda es dar calor para reducir el cólico menstrual, es el uso de alpiste, ya que el gel puede no cambia de estado al ser sometido al calor.

\begin{abstract}
This work shows a process of patterning and making a prototype panties with adaptation of thermal pads to reduce menstrual cramps adapted to the needs and requirements of women. There is talk of the transformation made for the preparation of the samples, from a basic template, as well as the 2 assembly processes of the same and the machinery used, in the same way the modifications made to the transformation are considered for better adaptation to the body in terms of cutting a garment and using materials. The results of the panties are shown with the adaptation of the compress bag according to the materials used for that purpose, where it is determined what is most convenient for the final use of the garment is to give heat to reduce menstrual colic, It is the use of birdseed, since the gel cannot change state when ever being in heat.
\end{abstract}

Citación: VÁZQUEZ-JUÁREZ, Yolanda, RAMÍREZ-MONDRAGÓN, Xóchitl y TENORIO-LARA, Raúl. Confección de un prototipo de pantaleta con adaptación de compresas térmicas para disminución de cólicos menstruales. Revista del Diseño Innovativo. 2019. 3-6: 1-7

\footnotetext{
* Correspondencia del Autor (Correo electrónico: yvazquezj@utsoe.edu.mx)

$\dagger$ Investigador contribuyendo como primer autor.
} 


\section{Introducción}

El objetivo de este proyecto es comprobar la funcionalidad del diseño de prototipo de pantaleta con adaptación de compresa térmica para cólico menstrual, a través de su confección con el fin de comprobar si la disminución del dolor en el vientre es efectiva al colocar las compresas en el área baja del vientre, dicho estudio se verificará en el uso de la prenda con una muestra de alumnas de la UTSOE

\section{Justificación}

El presente proyecto tiene la finalidad de crear una pantaleta que le ofrezca a las mujeres durante su ciclo menstrual un confort mediante la aplicación de calor en el bajo vientre, ya que al colocar las compresas tibias ayuda en la desinflamación y por consecuencia en la disminución de dolores abdominales.

\section{Problema}

Derivado de la primer etapa de investigación de este articulo donde se realizó el diseño de un prototipo de pantaleta con adaptación de compresas térmicas para disminución de cólicos menstruales, es necesario dar la continuidad a dicho proyecto, desde el procesos de patronaje y confección del mismo para verificar si los diseños son funcionales y cumplen su objetivo, ya que en la primer fase algunos fueron propuestos para ejercer presión adicional en el vientre sin considerar que puede afectar el uso de la compresa de gel al presionarla causando algún posible derrame del material que está hecha, así mismo se podrá comprobar si esa prenda es de utilidad para la disminución del cólico, pues será usada finalmente por algunas alumnas que presenten su ciclo menstrual al momento de realizar dicha prueba.

\section{Hipótesis}

El proporcionar calor en el abdomen ayuda a disminuir la presencia de cólicos menstruales.

\section{Objetivos}

\section{Objetivo General}

Confeccionar una pantaleta que permita la aplicación de presión y el uso de compresas térmicas con la finalidad de disminuir los cólicos menstruales en las alumnas de la UTSOE.

\section{Objetivos específicos}

- Patronar los diseños de los prototipos de las pantaletas.

- Confeccionar las muestras de los prototipos de las pantaletas.

- Comprobar el uso y función del diseño de las pantaletas, así como de los materiales seleccionados para dar calor.

\section{Marco Teórico}

\section{Marco Teórico}

\section{Dismenorrea}

La dismenorrea es el dolor intenso pélvico y abdominal que aparece en la mujer antes o durante la menstruación. La dismenorrea se caracteriza por provocar un dolor parecido al de un retortijón, pero más intenso, y puede llegar a acompañarse de náuseas, vómitos o mareos. Suele durar aproximadamente 24 horas y se estima que, aproximadamente un tercio de las mujeres tiene este tipo de periodo doloroso. Algunos estudios indican que la dismenorrea puede estar relacionada con un exceso de producción de prostaglandinas. Estas hormonas, que acentúan las contracciones de los músculos uterinos, podrían explicar el dolor intenso.

Aunque todas las mujeres pueden sufrir dismenorrea es más frecuente que aparezca en mujeres fumadoras, con sobrepeso, que han tenido la menarquía antes de los 11 años, o aquellas que consumen alcohol durante la menstruación.

En la actualidad este trastorno tiene una repercusión importante en la vida de la mujer ya que es causa de incapacitación laboral, consulta médica y automedicación.

\section{Causas}

Las causas de esta afección dependerán de si la mujer tiene dismenorrea primaria o secundaria En el caso de la primaria, las mujeres suelen tener contracciones uterinas anormales como consecuencia de un desajuste hormonal. En estos casos no hay una patología ginecológica que provoque el dolor. Las causas de la dismenorrea secundaria son otras patologías, como la endometriosis, los miomas uterinos, los quistes de ovario o las infecciones, entre otras. 


\section{Síntomas}

La manifestación principal de la dismenorrea es el dolor abdominal y/o pélvico. Además, otros síntomas que pueden tener las mujeres son:

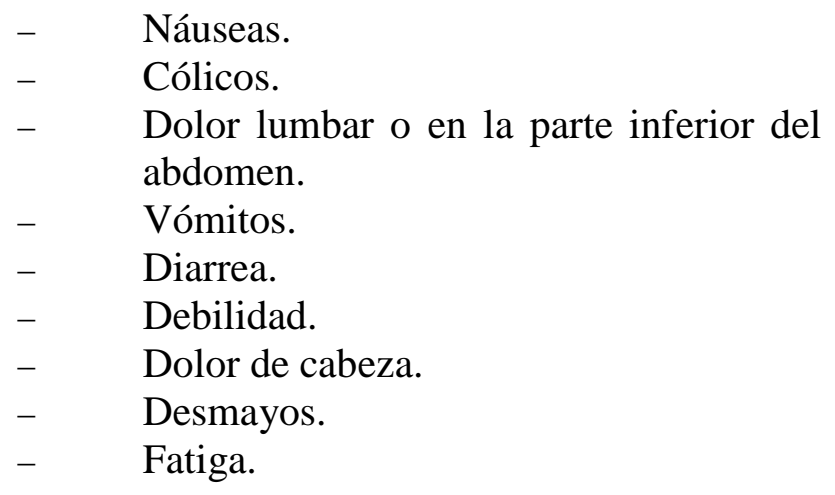

\section{Prevención}

La dismenorrea no se puede prevenir. Sin embargo, existen medidas que puede tomar la mujer para retrasar o reducir los síntomas.

Los especialistas recomiendan principalmente llevar una dieta equilibrada y realizar ejercicio físico de forma regular.

Otras medidas que puede tomar la mujer incluyen beber líquidos calientes, comer poco, pero frecuentemente, realizar masajes circulares en el abdomen, dormir de lado con las piernas dobladas, etc.

Además, medidas como la píldora anticonceptiva podrían reducir el dolor en algunas mujeres. No obstante, es recomendable que acuda a su ginecólogo.

Realizar deporte de forma regular puede ayudar a prevenir la aparición de los síntomas y reducir su intensidad

\section{Tipos}

Existen dos tipos de dismenorrea: primaria y secundaria.

\section{Dismenorrea primaria}

La dismenorrea primaria aparece como un dolor agudo en la zona baja del abdomen que comienza entre 45 y 24 horas antes del primer día del periodo. Este dolor suele desaparecer poco a poco durante el primer día de la menstruación.
Este tipo es frecuente entre las mujeres de 17 a 25 años de edad y no es frecuente en edades posteriores, especialmente si la mujer ha tenido hijos.

\section{Dismenorrea secundaria}

La dismenorrea secundaria consiste en el dolor agudo en la zona baja del abdomen, aunque, a diferencia de la primaria, éste es más pesado y continuo.

La secundaria suele manifestarse una semana antes de la menstruación y puede perdurar durante todo el periodo. Aparece principalmente en mujeres mayores de 30 años que han tenido hijos.

Una de las características de la dismenorrea secundaria es que puede ser un síntoma de otra enfermedad ginecológica o afección, como los miomas uterinos o la endometriosis.

\section{Tratamientos}

El tratamiento depende de varios factores y del tipo de dismenorrea.

Dismenorrea primaria: $\mathrm{Si}$ el dolor no es muy acusado y desaparece a lo largo del primer día de menstruación, los síntomas pueden aliviarse con algún anti inflamatorio, en algunas ocasiones, con tratamiento hormonal anticonceptivo. Se suele recomendar también realizar ejercicio de forma habitual, cambios en la dieta o hábitos de vida.

Dismenorrea secundaria: Si el dolor es más intenso, continuo y dura más tiempo es recomendable acudir al médico, quien determinará el tratamiento dependiendo de la causa y gravedad del dolor. Esto puede variar desde el tratamiento con analgésicos hasta una cirugía.

\section{Otros datos}

Cuando acudir al especialista

En los casos de dolor muy fuerte es preciso que la paciente consulte al médico, ya que puede requerir algún tipo de tratamiento hormonal. 
Esto es especialmente importante si la mujer no acostumbra a tener reglas dolorosas y de repente aparecen. En estos casos, la causa puede ser alguna alteración en el endometrio o por una enfermedad inflamatoria pélvica.

Algunas mujeres encuentran cierto alivio dándose un baño caliente y relajante o con un masaje relajante en el abdomen, en cuanto aparecen las primeras señales de dolor. (cuidate plus, 2019)

\section{Pantaleta}

a. Anote letras C-D en la cintura y letra E en el vértice de largo tiro y línea de tiro.

b. Prolongue la línea C-D hacia la derecha y escuadre $E$ hacia arriba hasta tocar la línea C-D (cintura), anote núm. 1 de 1 marque $1 \mathrm{~cm}$ hacia arriba y hacia abajo, núms. 1'y 1".

c. De $\mathrm{C}$ a la derecha marque $1 \mathrm{~cm}$ núm. 2

d. Prolongue la línea E-1 hacia abajo y maque la medida AVANCE TIRO (tabla No. 05, pág. 275) núm. 3.

e. $\quad$ En 3 escuadre $2.5 \mathrm{~cm}$ a la izquierda, núm. 4 de a escuadre una vertical con línea punteada que servirá como línea de apoyo.

f. Tome la medida 1-E, divídala a la mitad $\mathrm{y}$ de este punto escuadre una horizontal punteada a tocar el costado, núm. 5.

g. Una con línea punteada 4-5, tome la medida y divídala en tercios, anote $\mathrm{F}$ en el primer tercio.

h. Escuadre $\mathrm{F}$ hacia arriba y marque $6.4 \mathrm{~cm}$ para el calzón delantero, núm. 6 y $1 \mathrm{~cm}$ para el calzón trasero, núm. 7

Una los siguientes puntos:

Cintura: una 2-1'y 2-1"' con ligera curva

Costados: una 2-5 con curva sastre

Pierna delantera: una 5-6-4 con curva francesa pasando por la línea de apoyo en 4

Pierna trasero: una 5-7-4

Delantero: 1"'-2-5-7-4-3-E-1"

Trasero:1"'-2-5-7-4-3-E-1' (Couto, 2019)

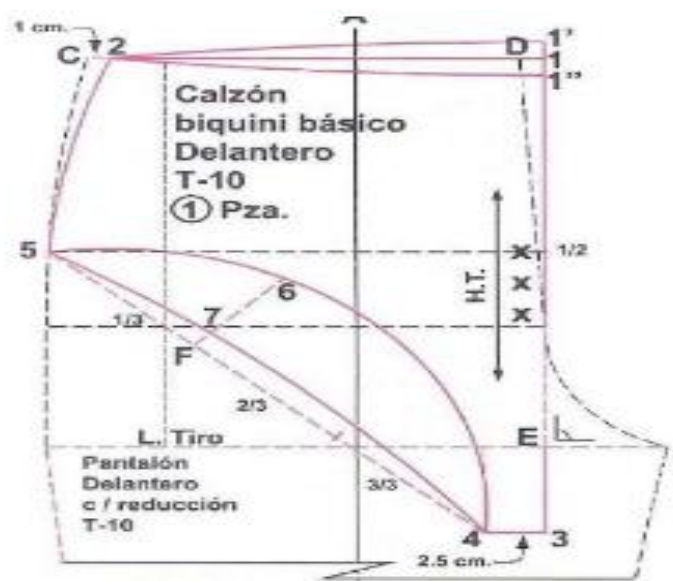

Figura 1

\section{Almohadilla o compresa}

La almohadilla o compresa de aromaterapia es un un cojín de semillas y hierbas elaborado en diferentes telas y estampados. Las almohadillas se usan en terapias de calor y frío. Contiene granos naturales y una mezcla de hierbas secas, que al tratarse en el calor o el frío emiten un efecto aromático relajante.

\section{Caliente}

Se puede calentar en el horno microondas. Disminuye los síntomas de Artritis, dolor de espalda, cólicos, nariz constipada, dolor muscular, dolor de cuello, entre otros.

\section{Frío}

Se puede enfriar en el congelador. Se utiliza para ojos inflamados, moretones, síndrome del túnel carpiano (entumecimiento y hormigueo en la mano y el brazo ocasionados por el pinzamiento de un nervio en la muñeca), fiebre, pies cansados, dolor de muelas, picaduras de insectos, estrés, dolor de cabeza y migraña. Por mencionar los más importantes.

Nota: al final encontrarás las preguntas más frecuentes del producto.

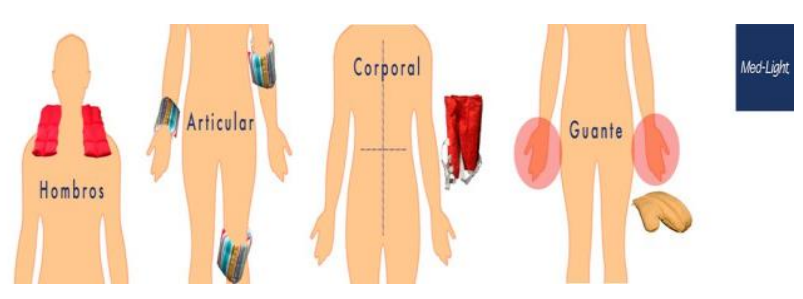

Figura 2 


\section{¿Cómo nos ayudan?}

Gracias a la acumulación de las semillas y hierbas que contiene más la temperatura deseada, el aroma que despiden ayuda a nuestro cuerpo de distintas formas. Por ejemplo, la manzanilla te ayuda a relajarte, el eucalipto a despejar las vías respiratorias, el zacate limón promueve una buena digestión, la flor de lavanda es relajante y ayuda contra las migrañas, la menta y la hierbabuena además de abrir las vías respiratorias, son relajantes y también benefician a la digestión y por último, recuerda que no son las únicas hierbas o semillas que pueden generar un beneficio a tu cuerpo, la canela además de ayudar contra las náuseas se le atribuyen propiedades antiinflamatorias y se ha utilizado contra el reumatismo.

\section{¿Para qué sirve una almohadilla o compresa?}

Literalmente es un remedio natural que se crea en múltiple diseño, formas y material la más versátil de todas es la almohadilla para cuerpo que puede ser utilizada casi en todo momento. Por ejemplo, se puede calentar y usar como relajante en el cuello, puede ser usada caliente sobre el vientre para cólicos, o bien para relajar los músculos durante o después de un entrenamiento sin importar el deporte, cuando tienes gripa la puedes usar sobre el pecho para despejar tus pulmones, etc. Ahora bien, si la enfrías puedes usarla para calmar dolores de cabeza, o en ocasiones para dolores dentales para bajar una inflamación. A temperatura ambiente puede ser usada como almohada anti estresante simplemente jugando con ella pues, de cualquier manera, el aroma que despide se percibe con solo manipularla. (medlightmedical, 2019)

\section{Metodología de la Investigación}

\section{Metodología de desarrollo del prototipo}

\footnotetext{
- Transformación

- Elaboración de pantaletas muestra, para realizar posibles adaptaciones en cuanto a su apariencia y ajuste.

- $\quad$ Corte y confección de la prenda final.

- $\quad$ Pruebas de uso de las pantaletas.
}

\section{Desarrollo}

\section{Transformaciones}

Se realizó el trazo de una pantaleta básica talla 32 , considerada por ser de talla comercial más usada, la primera transformación que se considero es con corte de pierna alta a la cintura, con 3 piezas, la segunda es pantaleta de pierna media a la cintura con 4 piezas en total.

\section{Elaboración de pantaletas muestra}

Se confeccionaron 2 prendas muestra que permitieran observar las mejoras a realizar en los patrones. La primera muestra se confecciono con 3 piezas: 1 delantero, 1 trasero y 1 puente, la confección se realizó de la siguiente manera:

\begin{tabular}{|c|c|c|}
\hline & Descripción & Máquina \\
\hline 1 & Unión de bases delantera y trasera & Over \\
\hline 2 & Colación de puente & Collareta \\
\hline 3 & Cerrar costados & Over \\
\hline 4 & Hacer dobladillos & collareta \\
\hline
\end{tabular}

Tabla 1 Ensamble de la primear muestra

La primera segunda muestra se confecciono con 4 piezas: 1 delantero, 1 trasero y 1 puente de tela y puente de algodón, la de confección fue de la siguiente forma:

\begin{tabular}{|l|l|l|}
\hline & Descripción & Máquina \\
\hline 1 & Unión de puente de tela y forro con delantero & over \\
\hline 2 & Unión de puente de tela y forro con trasero & over \\
\hline 3 & Cerrar costados & over \\
\hline 4 & Hacer dobladillos & collareta \\
\hline
\end{tabular}

Tabla 2 Ensamble de la segunda muestra

Tras el ensamble de las prendas se probaron para observar cual quedaba mejor en apariencia y determinar los cambios o adaptaciones a realizar. así mismo decidir la colocación del panel de contención de la compresa térmica. Por lo que se que se decidió que lo más conveniente en la prenda final es basarse en la confección de la segunda muestra, pero el patronaje a utilizar sería el de pierna alta haciendo ajuste con respecto a la altura de la cintura y el corte sería de 2 puentes igual a la transformación de la segunda muestra. 


\section{Corte y confección de la prenda final}

Para la confección de la prenda final algunos materiales por comodidad, así como el diseño original se adaptó, debido a que era necesario considerar la funcionalidad de la compresa térmica más que la presión ejercida en el vientre, por lo que en lugar de utilizar elástico o bandas de presión se trabajó con encaje elástico, que permitiera introducir con facilidad la bolsa del material térmico. El método de confección usado fue el mismo que se empleó en la segunda muestra, adecuando pasos a este proceso en la preparación la pieza delantera con el panel de encaje elástico donde se colocaría la compresa, así como entrepiernas y trasero (Tabla 3).

\begin{tabular}{|l|l|l|}
\hline & \multicolumn{1}{|c}{ Descripción } & Máquina \\
\hline 1 & Coser panel de encaje a delantero & collareta \\
\hline 2 & Unión de puente de tela y forro con delantero & over \\
\hline 3 & Unión de puente de tela y forro con trasero & over \\
\hline 4 & Coser encaje en entrepiernas y trasero & collareta \\
\hline 5 & Cerrar costados & over \\
\hline
\end{tabular}

Tabla 3 Ensamble de la pantaleta

Por otra parte, se confeccionaron dos bolsas con forma rectangular que se adaptaran a la pantaleta, una para el relleno de alpiste y otra para el gel.

\section{Resultados}

Al realizar la pantaleta se trabajaron algunas adecuaciones, como el alto de la pierna, el ancho de puente y bolsa de alpiste, la altura de la bolsa frontal se determinó en base a la segunda muestra (Figura 4., dando como resultado la siguiente pantaleta confeccionada (Figura 5).

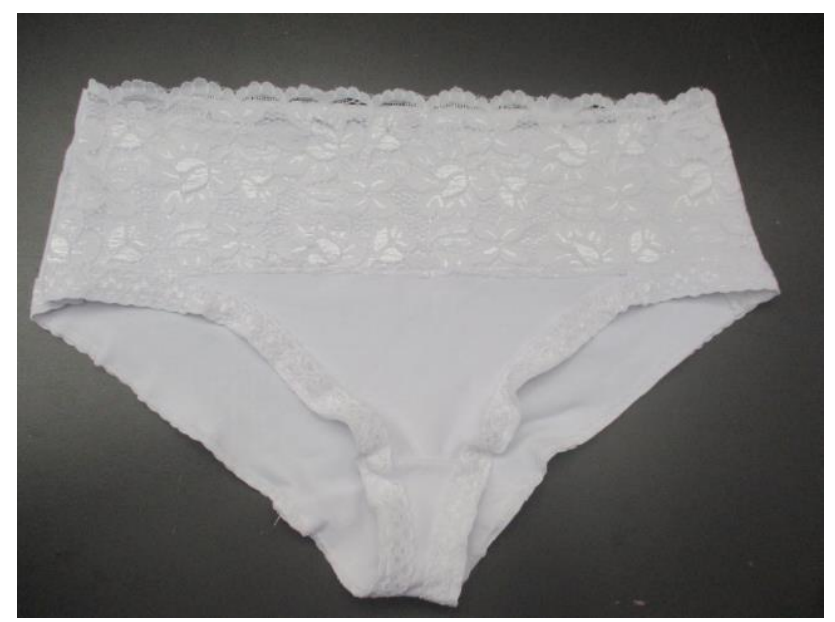

Figura 4 Pantaleta terminada con bolsa frontal

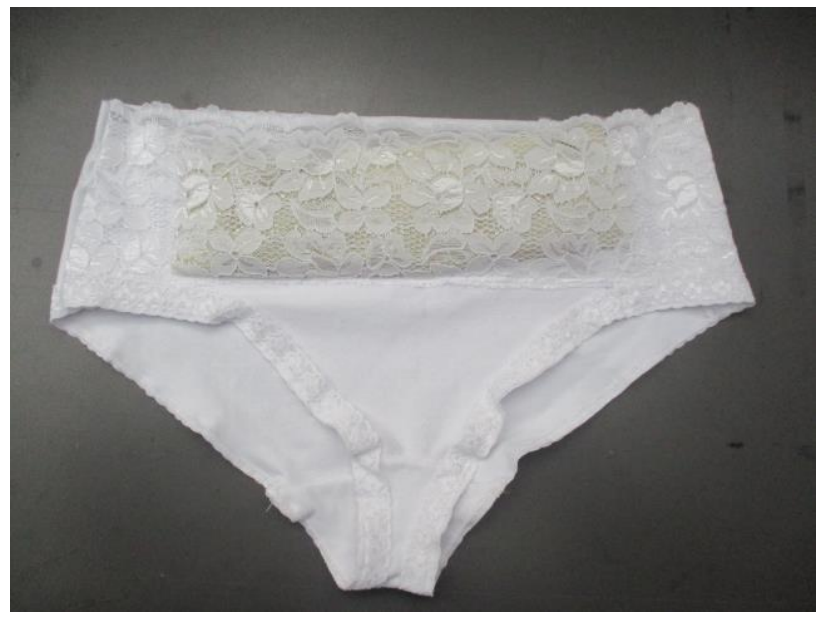

Figura 5 Pantaleta con bolsa de alpiste

Del análisis de los materiales de las almohadillas se consideró el alpiste, ya que guardaba por más tiempo el calor, además de dar una sensación de relajación por su textura. sin embargo, el gel al pasarse de la temperatura transfería exceso de calor (Figura 6).

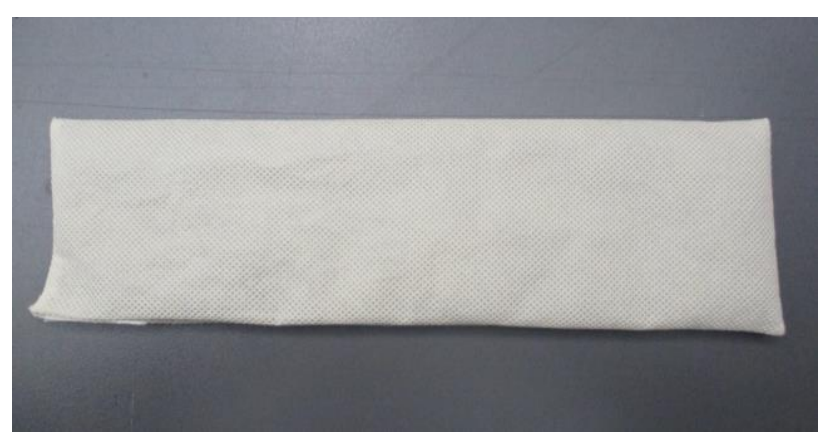

Figura 6 Almohadilla de alpiste

\section{Conclusiones}

- $\quad$ En primer lugar no debe aplicar la bolsa de gel directamente en la piel, sino que tendrá que utilizar un trapo o funda de por medio; el aplique directo en la piel podría ocasionar quemaduras y molestias. Tampoco debe dejarla en un lugar concreto durante mucho tiempo, pues esa zona podría resentirse. No debe presionarse de forma excesiva contra la piel, pues podríamos entorpecer la circulación y sus propiedades no serían útiles plenamente. Con el calor, se aliviarán dolores crónicos por lesiones subagudas.

Con respecto a la altura de la bolsa para la compresa no es relevante modificarla, pues esta prenda se puede escalar, por lo que cada usuaria seleccionara la talla adecuada a su cuerpo. 


\section{Referencias}

Couto, G. F. (2019). Libro $3^{\circ}$ : Aprenda Corte de ropa para Alta Costura Sistema "CyC". México D.F.: Couto y Confecciones S.A. de C.V.

cuidate plus. (2019). Obtenido de cuidate plus: https://cuidateplus.marca.com/enfermedades/gi necologicas/dismenorrea.html

medlightmedical. (2019). Obtenido de medlightmedical:

https://www.medlightmedical.com.mx/ 